\title{
A Hybrid CMOS APS Pixel for Wide-Dynamic Range Imaging Applications
}

\author{
Suat U. Ay \\ Department of Electrical and Computer Engineering, \\ University of Idaho, Moscow, ID 83844-1023 \\ Email: suatay@uidaho.edu
}

\begin{abstract}
In this paper, a new hybrid photodiode-photogate (HPDPG) CMOS APS pixel for high-dynamic range imaging applications is presented. The HPDPG pixel composes of a biased photogate, a photodiode and standard 3-transistors (3T) CMOS APS readout electronics. Biased photogate was connected in parallel with photodiode structure introducing a knee on the photoresponse curve. This new photodetection structure allows extension of the photoresponse range, providing wide dynamic range operation. New HPDPG pixel and a reference photodiode type 3 T CMOS APS pixel were designed, fabricated, and tested using a $0.5 \mu \mathrm{m}$ double poly, triple metal (2P3M) CMOS process. It was shown that addition of a biased photogate structure in a standard photodiode type 3T CMOS APS pixel improve saturation exposure five times $(5 x)$ with the expense of reduced quantum efficiency, and increased dark current. Photogate in parallel with photodiode improves pixel full-well capacity ten times $(10 x)$ with the expected reduction in sensitivity and conversion gain.
\end{abstract}

\section{INTRODUCTION}

Scenes we experience in our daily life have very wide range of light conditions from very bright to very dark. Regular image sensors are very good at capturing images in both of these scene conditions. However, we also experience scenes that have very large interscene dynamic ranges such as driving in and out of a dim tunnel during a bright sunny day. These scene conditions demand very wide dynamic range imagers. These light conditions pose more challenges for CMOS image sensors, which, in general, have lower dynamic range than that of the charge-coupled devices (CCD). Several approaches have been proposed to enhance the dynamic range of CMOS APS. One method is to add storage capacitor to the charge sensing node depending on the light condition, [1]. Another approach is sampling charge sensing node multiple times and constructing image afterwards using a fusion algorithms in digital domain [2]. Logarithmic response pixels have also achieved wide dynamic range operation with poor SNR and image quality [3][4]. Digital pixel sensors (DPS) that employs an ADC in each pixel provides improved dynamic range with the expense of area, [5][6]. These methods require either large number of transistors in each pixel increasing the imager size, or require extensive post processing of captured images, or provide poor image quality and/or speed.

In this work, a hybrid photodiode-photogate (HPDPG) CMOS APS pixel structure was proposed to extend the dynamic range of a standard photodiode (PD) type 3-transistor (3T) CMOS APS pixel. Dynamic range was extended by introducing a larger pixel capacitance after set threshold level. This added capacitance changes light response characteristics and sensitivity of the standard 3T photodiode APS pixel. A biased NMOS capacitor or photogate was used. Imaging capability of the NMOS capacitor was also utilized in the pixel by using fabrication process with non silicided poly gate. Proposed new pixel structure was fabricated along with a reference 3T photodiode APS pixel on same image sensor chip. Basic structure and operation principle of the HPDPG pixel is presented in section II. Design of the imager chip and pixels are explained in section III while measurement results are presented in section IV.

\section{Hypbid Photodiode-Photogate (HPDPG) Pixel}

\section{A. HPDPG Pixel Structure}

Cross sectional view of proposed CMOS hybrid photodiode-photogate (HPDPG) APS pixel is shown in Fig. 1. Control and readout electronics in the pixel is identical to standard 3T CMOS APS pixel. In HPDPG pixel, NMOS capacitor (NMOSCAP) is surrounded by the photodiode diffusion area which is also the source diffusion of the reset transistor. Gate of the NMOSCAP is connected to a global DC bias voltage. If the bias induced channel potential (Vchannel) of the NMOSCAP was set below the photodiode's reset level (Vreset), two different capacitances $(\mathrm{C} 1$, and $\mathrm{C} 2)$ appears in potential well diagram as depicted in Fig. 2. The capacitance $\mathrm{C} 1$ is associated with the active $\mathrm{n}+$ diffusions of the photodiode while $\mathrm{C} 2$ is related to the NMOSCAP. C2 becomes available when the photodiode voltage (Vpd) drops below the channel potential in where the NMOSCAP starts working in the saturation region.

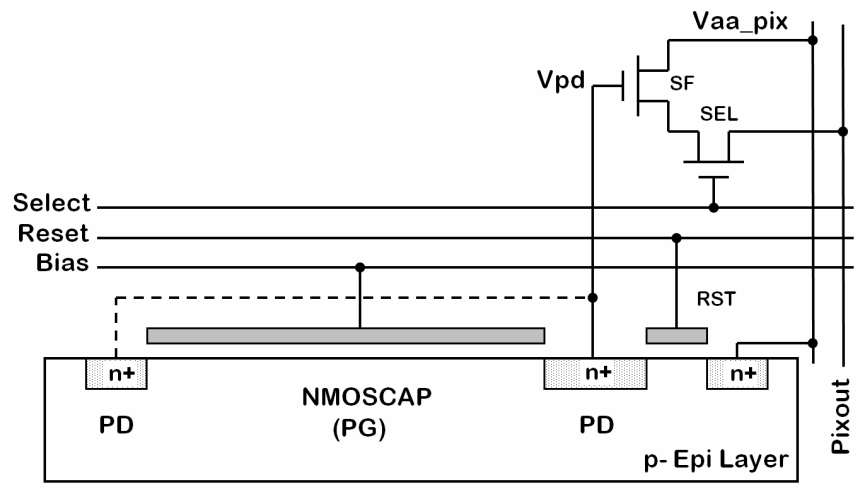

Figure 1. Cross section of proposed hybrid photodiode-photogate (HPDPG) CMOS APS pixel. 


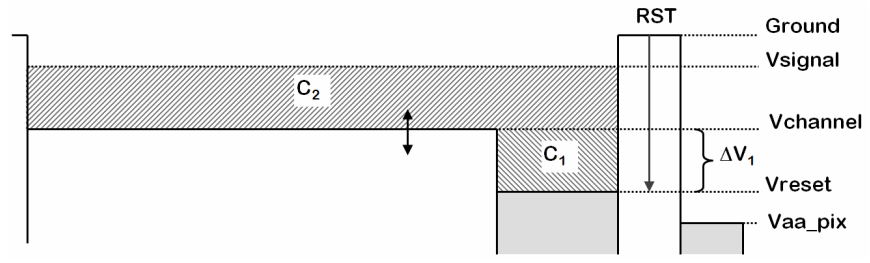

Figure 2. Ideal potential-well diagram of the HPDPG pixel.

Effect of NMOSCAP bias and consequently the channel voltage $\left(\mathrm{V}_{\text {channel }}\right)$ on light response characteristics of the HPDPG pixel are depicted in Fig. 3. If NMOSCAP bias reduced down to zero, photodiode pixel saturates at $\mathrm{L}_{\text {sat } 1}$ light level without seeing any extra capacitance. If pixel bias voltage increased, an extra "knee" $\left(1^{\text {st }}\right.$ knee $)$ appears on the response curve. This knee is modulated by the channel potential. Sensitivity of the HPDPG pixel is initially high, and the pixel capacitance is low (C1) until the first knee is reached. After the first knee, second capacitance (C2) is added to the total pixel capacitance decreasing the sensitivity. Second knee is reached when the signal chain saturates. The location of the second knee in terms of light exposure depends on the first knee location and the size of the second capacitance, or the size of the NMOSCAP. Higher exposure saturation is achieved by increasing the global bias voltage or by increasing the gate area of the NMOSCAP structure by design which results in a desired feature of wide dynamic range pixel operation. Since an NMOSFET structure was used in this design, higher the pixel NMOSCAP bias larger the pixel response range achieved. Opposite control behavior could be used for a PMOSCAP structure.

\section{B. Operation Principle of HPDPG Pixel}

Operation principle of HPDPG pixel is described on simulation setup shown in Fig.4. It composes of 3T APS pixel transistors (M1, M2, M3), column load transistor (M4), a biased NMOSCAP (PG), column parasitic sampling capacitance (CS), and a current source (Ipd) connected to photodiode node representing the photo current. A transient simulation was performed at 5.0 volt supply (VAA) and 3.5 volt pixel bias voltages (BIAS). Simulation result of different node voltages along with the normalized pixel capacitance is shown in the Fig.5. Pixel photodiode node was reset by activating the RST signal between 0.0 and $0.1 \mu \mathrm{sec}$. Vpd was reset to 3.5 volt. The threshold voltage of the reset transistor (M1) was about 1.5 volt because of the body effect. Same body effect can be observed on the PG transistor which is around 2.0 volt due to the larger device size. As soon as the

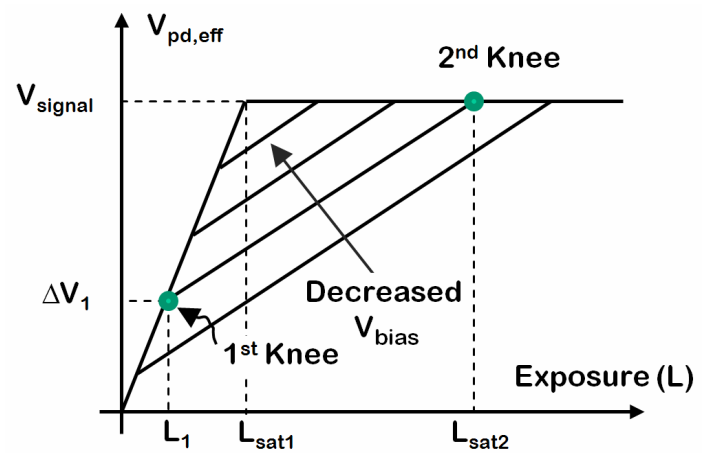

Figure 3. Light response characteristics of the HPDPG pixel.

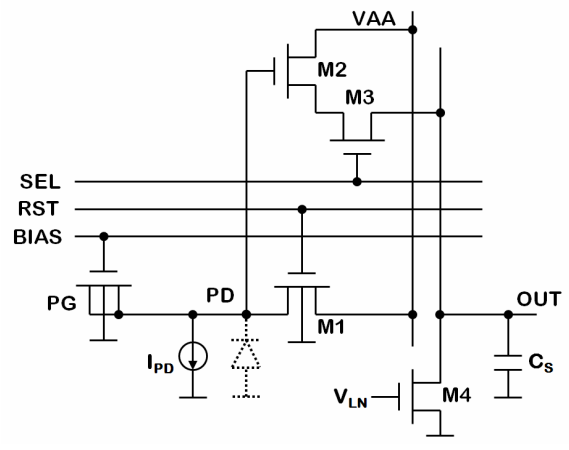

Figure 4. Simulation setup of HPDPG pixel.

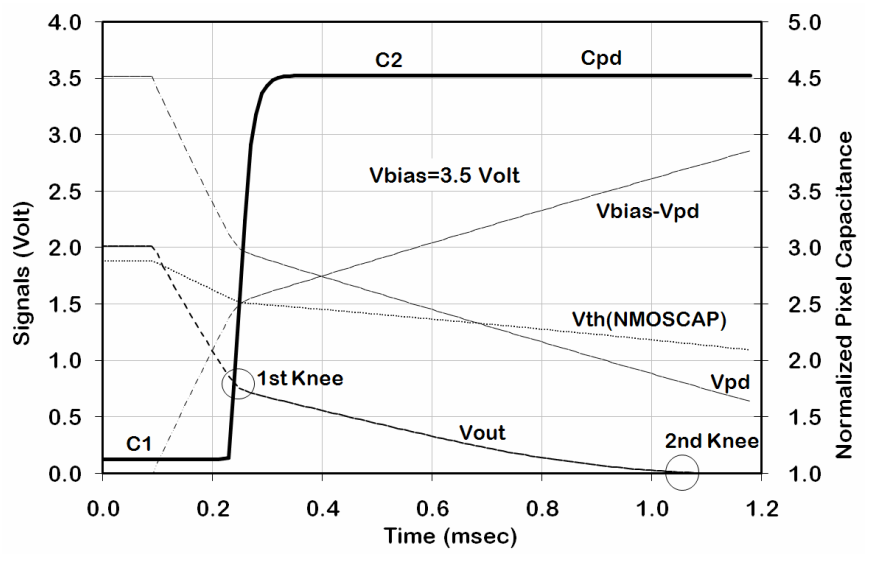

Figure 5. Simulation result at 3.5 volt pixel bias.

reset phase ends at $0.1 \mu \mathrm{sec}$, charge integration starts. The photo current (Ipd) discharges the predefined PD voltage. While Vpd drops, the overdrive voltage (Vbias-Vpd) of the PG increases. At the same time, the threshold voltage of the PG drops. Right after PD reset, the overdrive voltage is less than the threshold voltage, and the PG works in depletion mode. As a result, overall photodiode capacitance $(\mathrm{Cpd}=\mathrm{C} 1)$ is minimal. NMOSCAP starts to go into accumulation mode when the overdrive voltage became closer to the changing threshold voltage. Until this time, photodiode sensitivity is high because of the lower PD capacitance. As soon as strong inversion occurs on the $\mathrm{PG}$, pixel capacitance increases dramatically $(\mathrm{Cpd}=\mathrm{C} 2)$. This causes the sensitivity to drop and the first knee to appear on input-output curve (Vout), as shown in Fig. 5. Second knee appears when the pixel PD voltage moves below the input range of the pixel source follower.

\section{IMAGER AND PIXEL DeSIGN}

\section{A. Reference Pixel Design}

A reference photodiode (PD) pixel (REF2) was designed for performance comparison. Reference pixel layout and its cross section are shown in Fig.6a, and Fig. 7a. Photodiode region was made up of the $\mathrm{n}+$ diffusion and a donut shaped $\mathrm{N}$-Well region. It was placed at the edges of the $\mathrm{n}+$ diffusion to improve the collection efficiency of the photodiode and reduce overall PD capacitance in both pixels. Pixel pitch was chosen to be $18 \mu \mathrm{m}$. Total active photodiode area of the reference pixel (REF2) was $137 \mu \mathrm{m}^{2}$, resulting in a $42 \%$ pixel fill factor. 


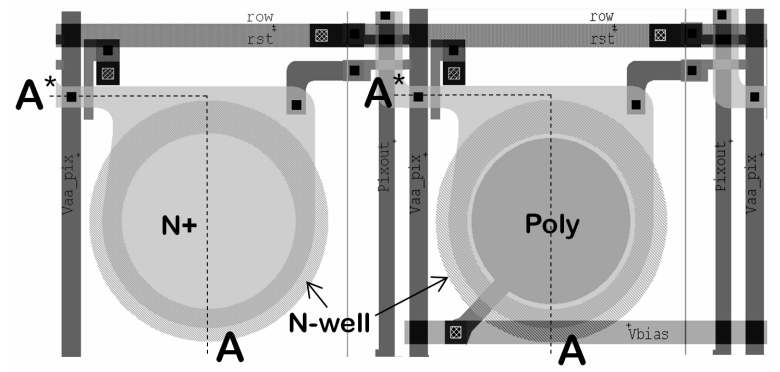

(a)

(b)

Figure 6. Layout of a) Reference (REF2) b) HPDPG pixels.

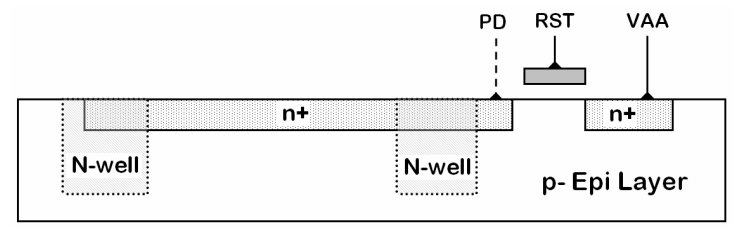

(a)



(b)

Figure 7. Layout cross section of a) Reference (REF2) b) HPDPG pixels.

\section{B. Hybrid Photodiode-Photogate (HPDPG) Pixel Design}

Proposed HPDPG pixel layout and its cross section view are shown in Fig.6b and Fig.7b, respectively. All imaging structures including the NMOSCAP poly-silicon gate were drawn circular. Bias was distributed only to the HPDPG pixels on the pixel array. In HPDPG pixel layout, circular PG area or the NMOSCAP gate poly occupies $58 \mu^{2}$ of total pixel area. Thus, pixel fill factor drops to $24 \%$. Although, PG contributes photon collection, it was expected to be minimal. Having large PG gate area reduces threshold variation enhancing the FPN performance of the pixel. This also helps to stabilize $1^{\text {st }}$ knee location.

\section{Imager Design}

A $0.5 \mu \mathrm{m}, 5$ Volt, double-poly, triple metal (2P3M) CMOS process with non silicide gate option was used for the design. A single serial readout architecture was adopted for reading pixel array, [7][8]. Pixel pitch was $18 \mu \mathrm{m}$. Imager composed of $424 \times 424$ pixel array, row decoder, row drivers, timing controls, digital and analog buffers, column analog signal processors (ASP), column decoder and multiplexer, and single serial readout channel. Pixel array was divided into 16 different subsections with 106 x 106 sub-arrays. In each subsection different pixel designs were placed. Reference and HPDPG pixels were placed on same columns, and read out through same column ASP and global readout circuitry. Microphotograph of the prototype imager is shown in Fig. 8.

\section{Measurement Results And Discussions}

Standard light and spectral measurements were performed on reference and HPDPG pixels under same environmental and imaging conditions.

\section{A. Conversion Gain and Full-Well Capacity Measurements}

Conversion gain of the reference and the HPDPG pixels were measured by using the photon transfer (PT) curve method, [9]. Measured PT curves for reference and HPDPG pixels at various bias conditions between 1 and 5 volts are shown in Fig.9. Two conversion gain factors were measured. First one is between dark and first knee start points. The second one is between transition end and second knee start points. The first one is associated with the pixel capacitance $\mathrm{C} 1$, and the second one is associated with $\mathrm{C} 2$. Conversion gain associated with the $\mathrm{C} 1$ could be adjusted between 4.6 and 2.0 $\mu$ Volt/e- for bias voltages between 0.1 and $5.0 \mathrm{~V}$. Conversion gain for $\mathrm{C} 2$ was measured at 4.0 and 5.0 volt bias voltages and they were 0.2 and $0.4 \mu$ Volt/e-. Overall, HPDPG pixel structure provides an order of magnitude full-well capacity improvement for standard 3T CMOS APS pixels in a given pixel size. At $5 \mathrm{~V}$ bias, 4 million electrons per pixel full-well capacity was achieved as shown in Fig. 10.

\section{B. Quantum Efficiency (QE) Measurement Results}

Quantum efficiency $(\mathrm{QE})$ of the reference (REF2) and the HPDPG pixels were measured, and the results are shown in Fig. 11. QE of the HPDPG pixel was measured at 0,3 , and 5 volt bias voltages. About $6.5 \%$ peak to peak quantum efficiency variation was observed between different bias voltages for the HPDPG pixel. Measured average peek quantum efficiency for HPDPG and reference pixels were $29.5 \%$ and $49.6 \%$ at $500 \mathrm{~nm}$, respectively. Average QE loss of HPDPG pixel compared to the reference pixel between 390 $\mathrm{nm}$ and $700 \mathrm{~nm}$ is $33.7 \%$.



Figure 8. Micrograph of Prototype CMOS APS imager chip

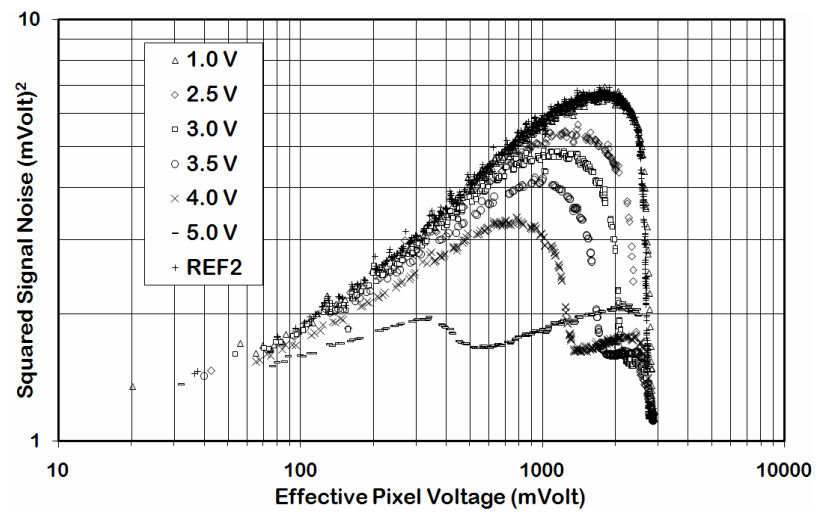

Figure 9. PT curves of REF2 and HPDPG pixels at different bias voltages. 


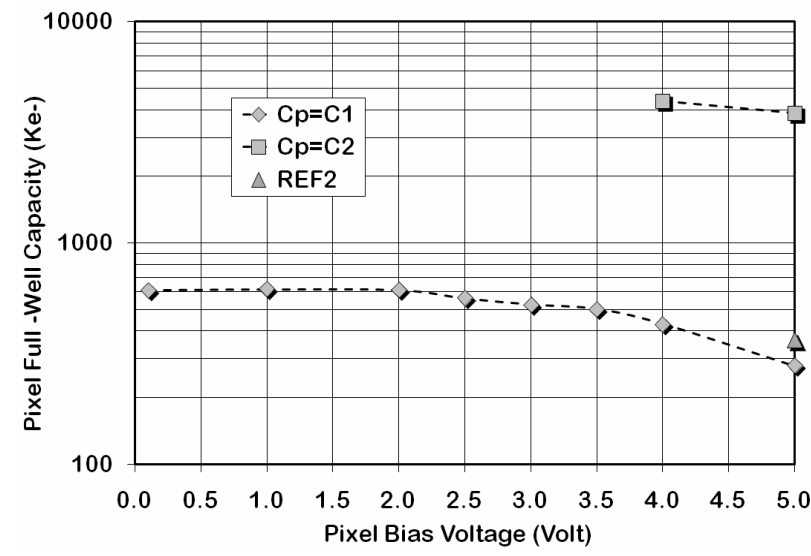

Figure 10. HPDPG Pixel full-well capacity associated with C1, and C2.

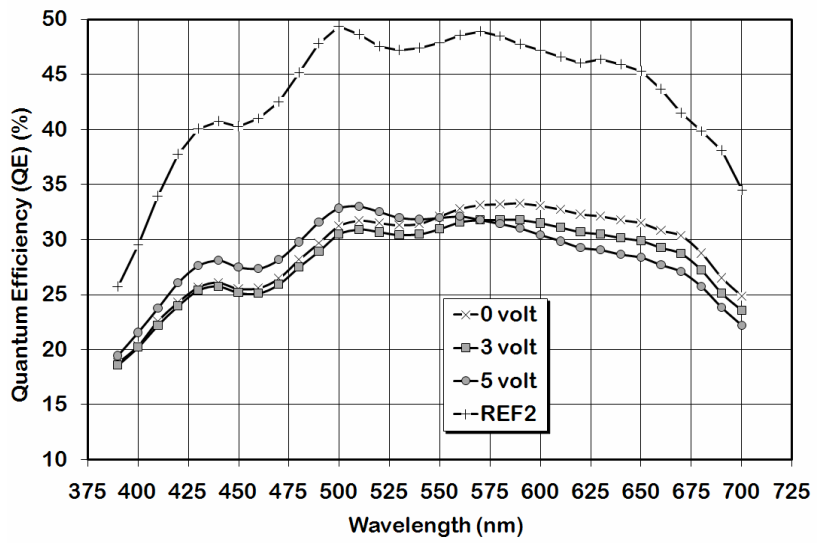

Figure 11. Quantum efficiency of reference (REF2) and HPDPG pixels.

\section{Linearity and Sensitivity Measurement Results}

Sensitivity and linearity of HPDPG pixel was measured for pixel biases of $0.1,3.0,4.0,4.5$ and 5.0 volts from pixel voltage versus exposure graphs shown in Fig 12. Bias dependent light response or dynamic range expansion for HPDPG pixel is clearly seen. As expected, first and second knee locations are also modulated with changing bias conditions. Sensitivity variation before the first knee is associated with the drop in the photodiode capacitance, $\mathrm{C} 1$. For increased pixel bias levels, this effect was observed on

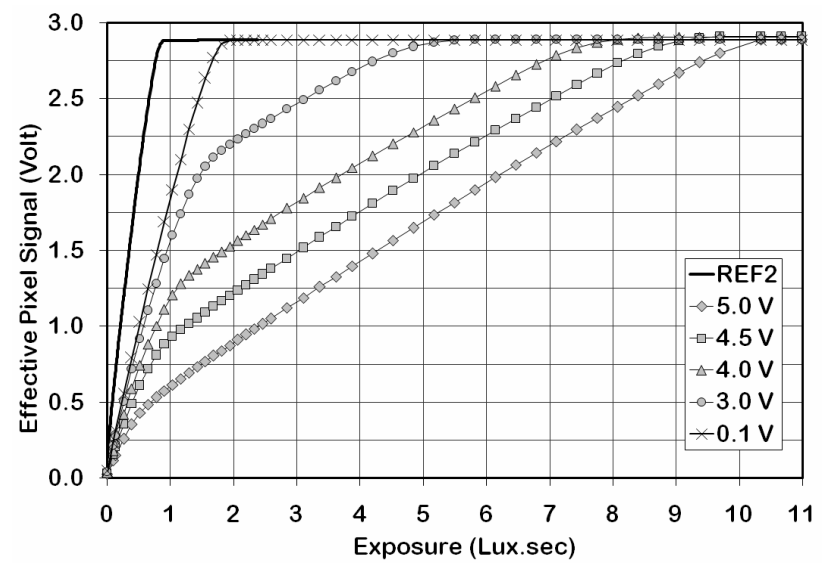

Figure 12. Measured sensitivity of the HPDPG and reference pixels. the photon transfer curves of the HPDPG pixel in Fig. 9. C1 related sensitivity varies between 1.9 and 0.8 Volt/Lux.sec, while the $\mathrm{C} 2$ related sensitivity variations were very little between 0.25 and 0.27 Volt/Lux.sec. Sensitivity of the reference (REF2) pixel was 3.75 Volt/Lux.sec. At 0.1 and 5 volt bias levels, HPDPG pixel cause sensitivity to drop two to five times, respectively. This is because of the increased pixel photodiode capacitance and reduced photodiode pixel fill factor about $45 \%$ for the HPDPG pixel. Fill factor of the photodiode region could be increased by placing the NMOSCAP device in the pixel that may be otherwise unused, such as under the routing metal lines. With this both sensitivity and the quantum efficiency could be improved.

\section{CONCLUSION}

A new CMOS pixel called hybrid photodiode-photogate (HPDPG) APS pixel was developed to improve the pixel fullwell capacity and dynamic range. HPDPG pixel composes of $\mathrm{n}+$ photodiode diffusion area and a biased NMOSCAP in each pixel. NMOSCAP was biased such a way that there are two knee appears in the light response curve. Locations of the knee points were adjustable with the pixel bias voltage that was connected to the gate of the NMOSCAP device. When the NMOSCAP was biased at strong inversion region by setting the bias voltage to 5 volt, more than 4 million electrons (or 10x improvement over reference pixel's full-well) full-well capacity was achieved at saturation while light response range was extended about 5 times providing wide-dynamic range pixel operation.

\section{ACKNOWLEDGMENT}

The author would like to thank Dr. E.R. Fossum and Micron Imaging characterization engineers for their guidance and technical support during this project.

\section{REFERENCES}

[1] S.Decker et al., "A 256x256 CMOS imaging array with wide dynamic range pixels and column parallel digital output," ISSCC Digest of Technical Papers, Feb.1998, pp.176-177.

[2] M.Schanz, C.Nitta, A.Bubmann, B.J.Hostika, and R.K.Wertheimer, "A high dynamic range CMOS image sensor for automotive applications," IEEE J. Solid State Circuits, vol.35, no.7, pp.932-938, Jul. 2000.

[3] C.Mead, AnalogVLSI and Neural Systems. Reading,MA:Addison

[4] Ricquier N., Dierickx B., "Pixel structure with logarithmic response for intelligent and flexible imager architectures". Microelectronics Engineering, vol. 19, pp. 631-634, 1992.

[5] D.X.D.Yang, A.ElGamal, B.Fowler, and H.Tian, "A640x 512 CMOS image sensor with ultra wide dynamic range floating-point pixel-level ADC," IEEE J. Solid-State Circuits, vol.34, no.12, pp.1821-1834, Dec.1999.

[6] J.Rhee, and Y.Joo, "Wide dynamic range CMOS image sensor with pixel level ADC," Electron.Lett.,vol.39,pp.360-361,Feb.2003.

[7] S. U. AY, M. Lesser, E. R. Fossum, "CMOS Active Pixel Sensor (APS) Image sensor for Scientific Applications”, Proc. of SPIE, vol. 4836, pp. 271-278, 2002.

[8] S.U. Ay, "Spectral response improvement of CMOS APS pixel through lateral collection," ISCAS, Dig. Of Tech. Papers, pp.1643-1646, 2006.

[9] J. R. Jenesick, Scientific Charge-Coupled Devices, SPIE Press, 2001 